\title{
Multiple infections with helminths - whipworm, hookworm, and roundworm
}
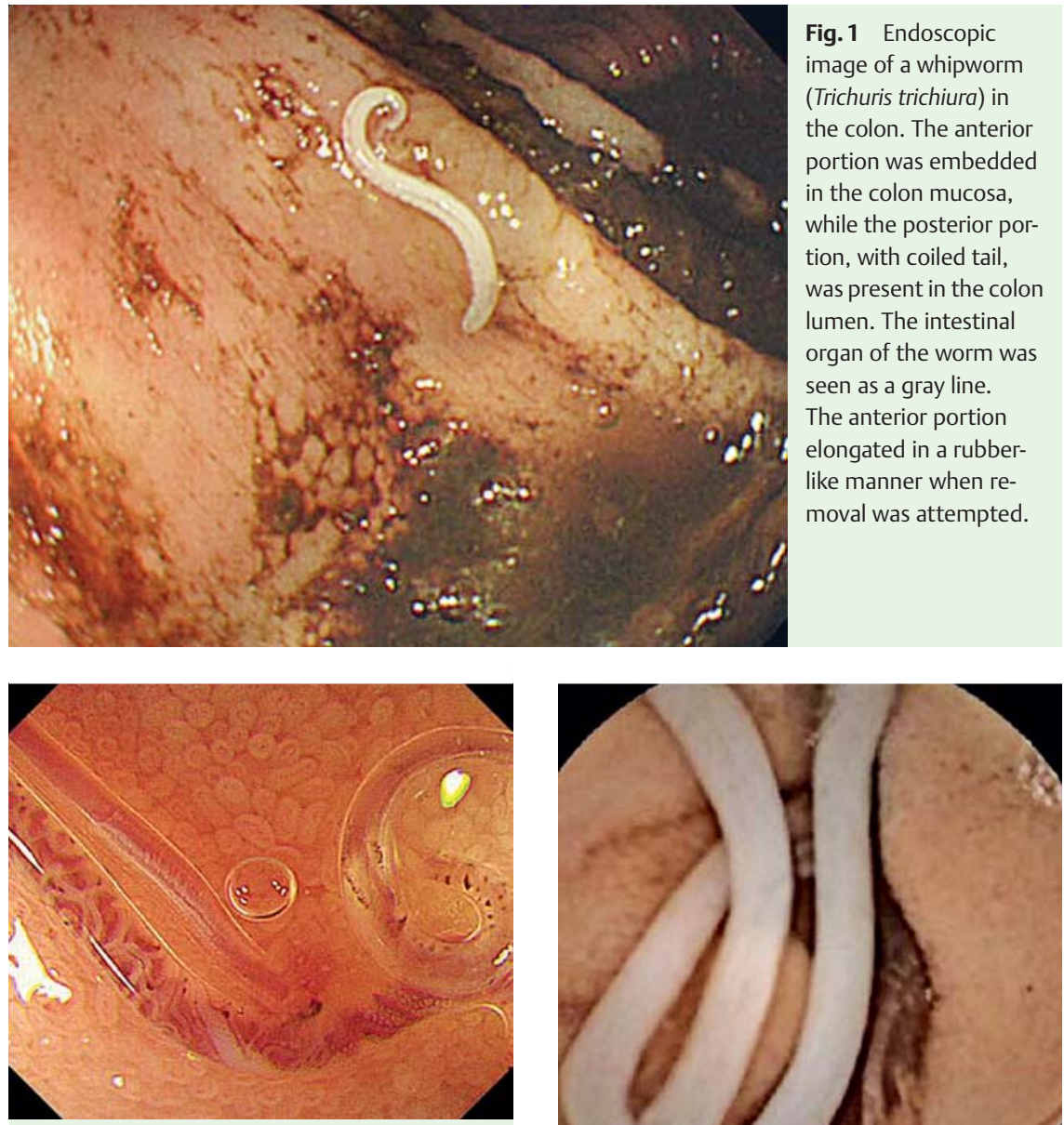

Fig. 3 Magnified endoscopic image of hookworms (Necator americanus) in the duodenum. The esophagus, intestines, and reproductive organs of the hookworm were clearly visible. The mouth was attached to the duodenal mucosa, with superficial mild erosion shown in red.

An 84-year-old woman presented with dyspnea due to an exacerbation of chronic heart failure caused by severe anemia (hemoglobin $4.4 \mathrm{~g} / \mathrm{dL}$ ). Colonoscopy revealed a cloud of white worms moving among tarry stool in the cecum and ascending colon. The posterior portions of the worms were located in the colon lumen, while the anterior portions were firmly embedded in colon mucosa ( $\bullet$ Fig. 1). One of the parasites was removed with biopsy forceps and identified as a whipworm (Trichuris trichiura).

Esophagogastroduodenoscopy (EGD) showed significant infection with threadlike worms, which were clinging to the duodenal mucosa and sucking blood, caus-

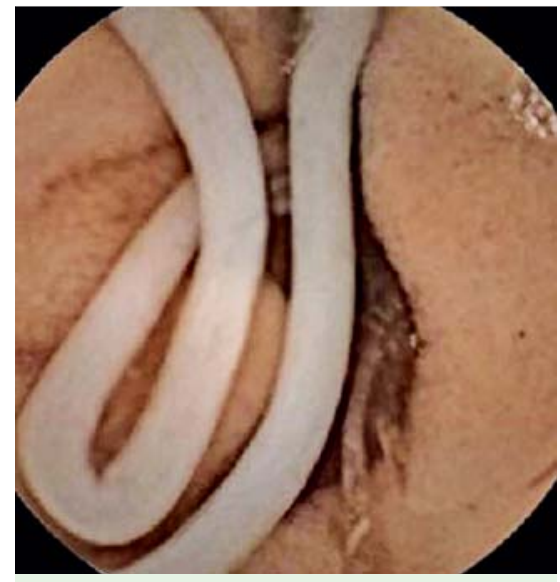

Fig. 4 Hookworms (Necator americanus) and a roundworm (Ascaris lumbricoides) were revealed on capsule endoscopy.

ing mild erosion ( $\bullet$ Fig. 2, Video 1 ). The intestinal and reproductive organs of the parasite were observed on magnified endoscopy ( Fig. 3). While observing these worms, a larger worm with a creamy white color was seen moving freely through the lumen ( Video 1 ). Both of these worms were retrieved using biopsy forceps; the

\section{Video 1}

Hookworms (Necator americanus) and a roundworm (Ascaris lumbricoides) on endoscopy. Several hookworms were seen clinging to the duodenal mucosa, while a roundworm moved freely through the lumen. Examples of both worms were retrieved using biopsy forceps.

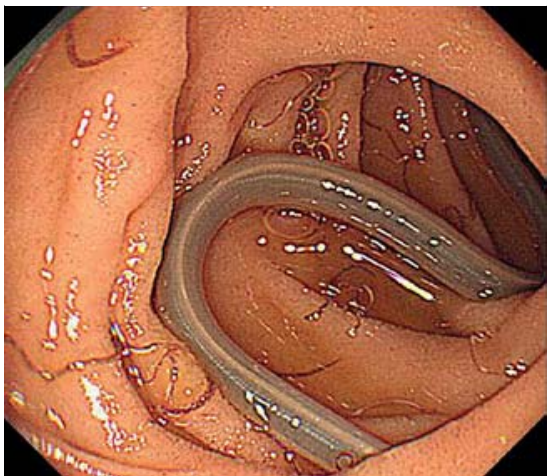

Fig. 2 Endoscopic images of hookworms (Necator americanus) and a roundworm (Ascaris lumbricoides). Several hookworms were seen clinging to the duodenal mucosa, while a roundworm moved freely through the lumen. Examples of both worms were retrieved using biopsy forceps.

smaller organism was found to be a hookworm (Necator americanus) and the larger one was a roundworm (Ascaris lumbricoides). Capsule endoscopy (Pillcam SB capsule; Given Imaging, Yoqneam, Israel) revealed both hookworm and roundworm infection in the small intestine. The hookworms were observed withdrawing blood from intestinal mucosa ( Fig.4, - Video 2).

Whipworms, hookworms, and roundworms are soil-transmitted helminths that present a major disease burden globally, but particularly in regions of poor sanitation. In this case, the entire gastrointestinal tract was examined, revealing that hookworms and roundworms infested the upper part of the small intestine, whereas whipworms lived in the large intestine. The patient was treated with a 3-day course of mebendazole. The eradication of parasites was confirmed on EGD and colonoscopy 2 weeks after completing pharmacotherapy. No eggs were found in a sub-

\section{Video 2}

Hookworms (Necator americanus) and a roundworm (Ascaris lumbricoides) were revealed on capsule endoscopy. The video clip was reconstructed from sequential images. In the small intestine, a roundworm and large numbers of hookworms were also evident. Mucosal bleeding was seen at the point where the hookworms were attached. 
sequent stool sample, and the patient had recovered from her anemia 1 month later.

Endoscopy_UCTN_Code_CCL_1AB_2AZ_3AZ

Competing interests: None
Kunimitsu Inoue ${ }^{1}$, Sotaro Ozaka', Kazuhisa Okamoto², Ryo Ogawa², Kazuhiro Mizukami², Tadayoshi Okimoto² $^{2}$ Masaaki Kodama², Takashi Kobayashi $^{3}$, Yuki Eshita ${ }^{3}$, Hideo Hasegawa ${ }^{4}$, Kazunari Murakami

${ }^{1}$ Department of Gastroenterology, Almeida Hospital, Oita, Japan

${ }^{2}$ Department of Gastroenterology, Faculty of Medicine, Oita University, Yufu, Japan ${ }^{3}$ Department of Infection Control, Faculty of Medicine, Oita University, Yufu, Japan

${ }^{4}$ Department of Biology, Faculty of Medicine, Oita University, Yufu, Japan
Bibliography

DOI http://dx.doi.org/

10.1055/s-0034-1364887

Endoscopy 2014; 46: E117-E118

(c) Georg Thieme Verlag KG

Stuttgart · New York

ISSN 0013-726X

\section{Corresponding author}

Kunimitsu Inoue, MD

Department of Gastroenterology Almeida Hospital

1509-2 Miyazaki

Oita, 870-1195

Japan

Fax: +81-97-5680743

kuninoue@oita-u.ac.jp 